\title{
Tabela de vida de fertilidade de Lysiphlebus testaceipes (Cresson, 1880) (Hymenoptera, Aphidiidae) em Schizaphis graminum (Rondani, 1852) (Hemipera, Aphididae)
}

\author{
Sandra Maria Morais Rodrigues ${ }^{1}$ \\ Vanda Helena Paes Bueno ${ }^{1}$ \\ Marcus Vinicius Sampaio ${ }^{1}$
}

\begin{abstract}
Fertility life table of Lysiphlebus testaceipes (Cresson, 1880) (Hymenoptera, Aphidiidae) on Schizaphis graminum (Rondani, 1852) (Hemipera, Aphididae). Life table analyses have been developed to understanding the impact of various sources of intrinsic and extrinsic mortalities on the rate of population growth. The understanding of the population increase of the parasitoids related to their hosts is important in biological control programs. This work had as objective to evaluate the survival and fertility of the parasitoid Lysiphlebus testaceipes (Cresson, 1880) on Schizaphis graminum (Rondani, 1852) as a host under fertility life table. The experiment were carried out in a climatic chamber at $25 \pm 1{ }^{\circ} \mathrm{C}, \mathrm{RH}$ $60 \pm 10 \%$ and $10 \mathrm{~h}$ photophase. To determine the immature mortality, the development time and the sex ratio of the parasitoid, 12 females of the parasitoid (less than one day old) and 240 nymphs of $S$. graminum (3 days old) were used. To evaluate the longevity and fertility of $L$. testaceipes, 15 females (less than one day old) were used. Nymphs of $S$. graminum ( 3 days old) were offered for each parasitoid female daily, until the female died, being in the $1^{\text {st }} d a y-300$ nymphs; $2^{\text {nd }}$ day - 250 nymphs; $3^{\text {rd }}$ day - 200 nymphs; $4^{\text {th }}$ day - 150 and in the other days a number of 50 nymphs. L. testaceipes had an immature mortality of $22,2 \%$, and a development time of males and females of 9.0 and 9.1 days, respectively. The females of L. testaceipes laid, in it first life day, $257.8 \mathrm{eggs}$, and they survived up until seven days. The net reproduction rate $\left(\mathrm{R}_{\mathrm{o}}\right)$ and the intrinsic rate of increase $\left(\mathrm{r}_{\mathrm{m}}\right)$ were respectively, 301.9 and 0.513 . The finite rate of increase (1) was 1.67 females per day, the mean length of a generation (T) was 11.13 days and the time to duplicate the population (TD) was 1.35 weeks. The parasitoid L. testaceipes have a high potential of population growth on $S$. graminum as a host under the analyzed conditions.
\end{abstract}

KeYwords. Fertility table; Lysiphlebus; parasitoid; reproduction rate.

\section{INTRODUÇÃO}

A determinação de tabelas de vida auxilia tanto na compreensão da dinâmica populacional de uma espécie como na avaliação do impacto que inimigos naturais podem causar sobre a população de uma determinada praga (VAN LENTEREN \& Woets 1988; Bellows-JUNIOR et al. 1992). O crescimento de uma população é estimado com base em dados de sobrevivência e fertilidade que são sintetizadas em tabelas denominadas tabelas de vida de fertilidade.

$\mathrm{Na}$ natureza, um ou vários fatores podem predominar e influenciar a razão real de aumento (r) de um inseto. Porém, em condições de laboratório é possível excluir esses fatores e, assim, determinar a taxa intrínseca de aumento $\left(\mathrm{r}_{\mathrm{m}}\right)$. Esta taxa é definida como a máxima razão de aumento obtido por uma população de distribuição etária fixa, em qualquer combinação particular dos fatores físicos do tempo, em condições ótimas de espaço, alimentação e sem a influência de outros fatores. No entanto, o valor da taxa intrínseca de aumento $\left(\mathrm{r}_{\mathrm{m}}\right)$ não será o mesmo para climas e fontes de alimento diferentes (ANDREWARTHA \& BIRCH 1954).

Dentre os diversos critérios de seleção e avaliação de inimigos naturais, um agente de controle biológico será considerado efetivo contra uma determinada praga se, pelo menos, as taxas intrínsecas de aumento $\left(\mathrm{r}_{\mathrm{m}}\right)$ de ambos forem semelhantes e, neste caso, é necessário que introduções regulares sejam feitas para que o controle desejado seja obtido (VAN LENTEREN 1986).

O parasitóide afidí́deo, Lysiphlebus testaceipes (Cresson, 1880), é tido como um agente de controle biológico promissor

1. Departamento de Entomologia, Universidade Federal de Lavras. Caixa Postal 37, 37200-000 Lavras-MG, Brasil.

Endereço eletrônico: smmrodrigues@hotmail.com; vhpbueno@ufla.br; marcsampaio@yahoo.com.br 
para diversas espécies de pulgões, como Schizaphis graminum (Rondani, 1852) e Aphis gossypii Glover, 1877 (BERGMANn et al. 1996; Elliot et al. 1999). Em estudos realizados por RoDRIGUES \& Bueno (2001), S. graminum e A. gossypii mostraram-se adequados para o desenvolvimento de $L$. testaceipes, com taxas de parasitismo de $76 \%$ e $56 \%$, respectivamente, e emergência de $100 \%$ e $83 \%$, respectivamente. Quando L. testaceipes foi mantido em $A$. gossypii a $25^{\circ} \mathrm{C}$, apresentou uma taxa intrínseca de aumento $\left(\mathrm{r}_{\mathrm{m}}\right.$ ) de 0,400 e longevidade média de 2,6 dias ( VAN STEENIS 1994).

Embora L. testaceipes apresente um amplo espectro de hospedeiros, informações quanto à sua reprodução em hospedeiros adequados são escassas. Dessa forma, este trabalho teve por objetivo estimar a sobrevivência e fertilidade de L. testaceipes, tendo como hospedeiro $S$. graminum, por meio de uma tabela de vida de fertilidade.

\section{MATERIAL EMÉTODOS}

O potencial de crescimento do parasitóide, L. testaceipes, foi determinado em uma câmara climatizada a $25 \pm 1^{\circ} \mathrm{C}, 60 \pm 10 \%$ de umidade relativa e $10 \mathrm{~h}$ de fotofase. Foram analisados os seguintes parâmetros: mortalidade de imaturos, desenvolvimento, reprodução e longevidade das fêmeas. Indivíduos do parasitóide, L. testaceipes e de S. graminum, foram obtidos de criações existentes no Laboratório de Controle Biológico do Departamento de Entomologia da Universidade Federal de Lavras (UFLA).

Mortalidade de imaturos e desenvolvimento de $\boldsymbol{L}$. testaceipes em S. graminum. Para a determinação da mortalidade de imaturos e desenvolvimento de L. testaceipes, foram utilizadas 12 fêmeas do parasitóide com menos de um dia de idade, acasaladas, alimentadas com solução de mel a $20 \%$, e 240 ninfas de $S$. graminum com três dias de idade.

Vinte ninfas do pulgão foram colocadas em placas de Petri (6 cm de diâmetro) contendo um disco foliar de sorgo (Sorghum bicolor L., variedade BR303) em uma camada de ágar-água (1\%). Após a primeira oviposição, a fêmea de L. testaceipes ficou em contato com os indivíduos hospedeiros por dez minutos. Após o parasitismo, dez ninfas de $S$. graminum foram retiradas das placas e individualizadas em outras placas de Petri ( $6 \mathrm{~cm}$ de diâmetro) contendo uma camada de ágar-água ( $1 \%$ ) e um disco foliar de sorgo ( $3 \mathrm{~cm}$ de diâmetro) e mantidas em câmara climática. Foram avaliados o dia da mumificação, a emergência e a razão sexual dos parasitóides. As outras dez ninfas de $S$. graminum foram dissecadas após três dias do parasitismo, para efetuar a contagem das larvas vivas e/ou mortas no interior das ninfas hospedeiras, estimando-se a mortalidade de imaturos do parasitóide.

Reprodução e longevidade de $L$. testaceipes em $S$. graminum. Fêmeas ápteras de $S$. graminum foram colocadas em unidades de criação semelhantes às usadas por RODRIGUES \& BuENo (2001) e a cada $24 \mathrm{~h}$ as ninfas nascidas foram transferidas para outra unidade de criação. Quando as ninfas

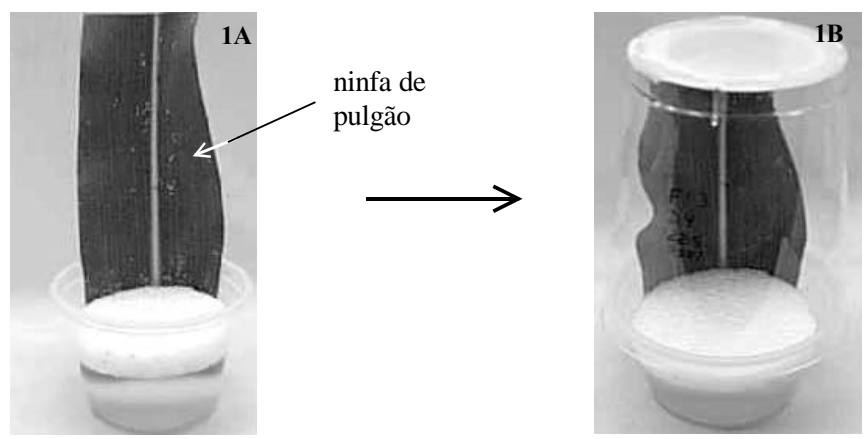

Fig. 1. Recipiente utilizado para observações da reprodução de Lysiphlebus testaceipes tendo como hospedeiro Schizaphis graminum.

estavam com três dias de idade foram submetidas ao parasitismo por fêmeas de L. testaceipes. Isto foi feito para que todas as fêmeas parasitóides que emergissem tivessem o mesmo tamanho, uma vez que segundo HÅgVAR \& Hofsvang (1991) a fecundidade é influenciada pelo tamanho da fêmea. Quinze fêmeas do parasitóide com menos de um dia de idade foram, então, acasaladas, alimentadas com solução de mel a $20 \%$ e utilizadas no experimento.

A unidade experimental, para observações quanto à reprodução de L. testaceipes, foi constituída por um copo plástico $(100 \mathrm{~mL})$ contendo água destilada e uma folha de sorgo fixada por um disco de isopor (Fig. 1A). Para isolamento desta folha foi colocado um copo plástico $(250 \mathrm{~mL})$ com a abertura para baixo sobre o copo plástico de $100 \mathrm{~mL}$ (Fig. 1B). A aeração no recipiente foi favorecida por uma abertura ( $4 \mathrm{~cm}$ de diâmetro) no fundo do copo de $250 \mathrm{~mL}$, que foi coberta por organza (Fig. 1B). Em cada recipiente existia uma colônia com ninfas de $S$. graminum de três dias de idade e uma fêmea de L. testaceipes com menos de um dia de idade. Até a morte da fêmea parasitóide, foi oferecida diariamente, uma nova colônia de S. graminum.

Os recipientes contendo as ninfas parasitadas permaneceram a $25^{\circ} \mathrm{C}$ por três dias, para que houvesse o desenvolvimento das larvas do parasitóide. Após esse período, as ninfas parasitadas foram acondicionadas em um freezer com o intuito de paralisar o desenvolvimento das larvas e, assim, evitar que houvesse uma competição intraespecífica, no interior do hospedeiro, e conseqüentemente, a eliminação de larvas supernumerárias. A estimativa da quantidade de ovos que as fêmeas parasitóides haviam colocado nas ninfas de $S$. graminum foi obtida através da dissecação dessas ninfas em uma solução de cloreto de sódio (1\%), com o auxílio de estiletes, sob microscópio estereoscópico. Essa metodologia utilizada, quanto à contagem de ovos, seguiu aquela proposta por VAN SteEnis (1994), onde o número de larvas encontrado correspondeu ao número de ovos no interior do hospedeiro.

As densidades de ninfas hospedeiras utilizadas durante o período de vida das fêmeas parasitóides foram: $1^{\circ}$ dia - 300 ninfas; $2^{\circ}$ dia - 250 ninfas; $3^{\circ}$ dia - 200 ninfas; $4^{\circ}$ dia -150 e nos demais dias um número de 50 ninfas. 
Análise dos dados. De acordo com os dados obtidos elaborou-se a tabela de vida de fertilidade de $L$. testaceipes em S. graminum, baseando-se em ANDREWARTHA \& BIRCH (1954), onde $\mathbf{x}$ é o ponto médio de cada idade das fêmeas parentais, contada a partir da fase de ovo; $\mathbf{l}_{\mathbf{x}}$, expectativa de vida até a idade $\mathbf{x}$, expressa como uma fração de uma população inicial de uma fêmea; $\mathbf{m}_{\mathbf{x}}$ fertilidade específica, ou seja, o número de descendentes produzidos por fêmea na idade $\mathbf{x}$ e que originarão fêmeas; $\mathbf{l}_{\mathbf{x}} \mathbf{m}_{\mathbf{x},}$ o número total de fêmeas nascidas na idade $\mathbf{x}$.

Os parâmetros de crescimento da população do parasitóide resultantes da tabela de vida também foram calculados segundo Andrewartha \& Birch (1954); onde $\mathrm{R}_{\mathrm{o}}$ é a taxa líquida de reprodução, ou seja, a taxa de aumento populacional, considerando fêmeas de uma geração para outra; MGT ou T (tempo médio de geração), duração média de uma geração; $r_{m}$, capacidade inata de aumentar em número); $\lambda$, razão finita de aumento, ou seja, é o número de vezes em que a população multiplica em uma unidade de tempo; DT, tempo que leva a população para duplicar em número.

\section{RESULTADOS EDISCUSSÃO}

Mortalidade de imaturos e desenvolvimento de $\boldsymbol{L}$. testaceipes em S. graminum. Das ninfas de S. graminum dissecadas, após exposição ao parasitismo por fêmeas de $L$. testaceipes, foi constatado que, em cerca de 14,5\% das mesmas, não havia larvas do parasitóide. VAN STEENIS (1994) utilizando o hospedeiro $A$. gossypii, a $25^{\circ} \mathrm{C}$, observou $19,7 \%$ de pulgões sem larvas de $L$. testaceipes.

A mortalidade de imaturos de L. testaceipes foi de $22,2 \%$, sendo que $10,4 \%$ correspondeu à mortalidade larval e $11,8 \%$ à mortalidade pupal (Tabela I). Este valor foi inferior ao encontrado por VAN STEENIS (1994) $(29,6 \%)$ quando $L$. testaceipes teve como hospedeiro A. gossypii a $25^{\circ} \mathrm{C}$.

Quanto mais adequado for um hospedeiro para o desenvolvimento de um parasitóide, menor será a mortalidade das formas jovens do mesmo. Segundo MACKAUER et al. (1996), as fêmeas de parasitóides da família Aphidiidae ao encontrarem

Tabela I. Mortalidade de imaturos e desenvolvimento de L. testaceipes em $S$. graminum a $25 \pm 1{ }^{\circ} \mathrm{C}, 60 \pm 10 \%$ de umidade relativa e 10 horas de fotofase.

\begin{tabular}{lcc}
\hline Caráter avaliado (\%) & Média \pm EP* & $\begin{array}{c}\text { Número de } \\
\text { indivíduos (n) }\end{array}$ \\
\hline $\begin{array}{l}\text { Pulgões sem larva no } \\
\text { momento da dissecação }\end{array}$ & $14,5 \pm 2,89$ & 16 \\
Mortalidade larval & $10,4 \pm 3,28$ & 12 \\
Mortalidade pupal & $11,8 \pm 4,02$ & 12 \\
mortalidade total de imaturos & $22,2 \pm 4,59$ & 24 \\
\hline Ciclo de desenvolvimento & & \\
(dias) & & \\
\hline Machos & $9,0 \pm 0,11$ & 33 \\
Fêmeas & $9,1 \pm 0,05$ & 47 \\
\hline
\end{tabular}

*Erro padrão da média

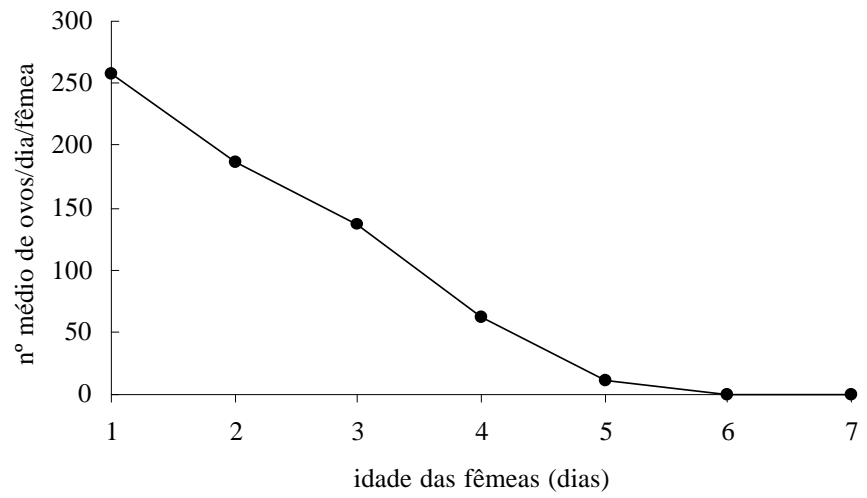

Fig. 2. Produção diária de ovos por fêmea de Lysiphlebus testaceipes em função da idade, a $25 \pm 1{ }^{\circ} \mathrm{C}, 60 \pm 10 \%$ de umidade relativa e 10 horas de fotofase.

um hospedeiro potencial analisam-no usando as antenas e o ovipositor e esse hospedeiro só receberá um ovo se apresentar características fisiológicas e nutricionais mínimas que permitam o desenvolvimento das suas formas jovens.

O tempo médio de desenvolvimento para machos e fêmeas de L. testaceipes, foi 9,0 e 9,1 dias, respectivamente (Tabela I). Valores semelhantes foram obtidos por VöLKL et al. (1990), quando esse afidiídeo ovipositou em Pentalonia nigronervosa Coquerel, 1859 a $24^{\circ} \mathrm{C}$, apresentando um tempo médio de desenvolvimento de 8,9 dias para machos e 9,5 dias para fêmeas. A razão sexual para $L$. testaceipes em $S$. graminum foi de 0,6 e foi semelhante àquela observada por KRING \& KRING (1988) para esse mesmo parasitóide, 0,66.

Tabela de vida de fertilidade de $L$. testaceipes em $S$. graminum. Fêmeas de L. testaceipes não apresentaram um período de pré-oviposição, uma vez que no primeiro dia de vida ovipositaram 257,8 ovos nas ninfas hospedeiras de $S$. graminum, o que correspondeu ao pico de produção de descendentes. Já VAN STEENIS (1994), observou cerca de 120 ovos de L. testaceipes em A. gossypii a $25^{\circ} \mathrm{C}$, no primeiro dia de vida desse parasitóide.

Tabela II. Fecundidade diária de Lysiphlebus testaceipes em Schizaphis graminum a $25 \pm 1^{\circ} \mathrm{C}, 60 \pm 10 \%$ de umidade relativa e 10 horas de fotofase.

\begin{tabular}{ccccc}
\hline $\begin{array}{c}\text { Idade } \\
\text { da fêmea } \\
\text { (dias) }\end{array}$ & $\begin{array}{c}\text { Número } \\
\text { de } \\
\text { fêmeas }\end{array}$ & $\begin{array}{c}\text { Pulgões } \\
\text { oferecidos/ } \\
\text { fêmea }(\mathrm{n})\end{array}$ & $\begin{array}{c}\text { Total de } \\
\text { ovos/fêmea } \\
(\overline{\mathrm{X}} \pm \mathrm{EP} *)\end{array}$ & $\begin{array}{c}\text { Pulgões não } \\
\text { parasitados } \\
(\overline{\mathrm{X}} \pm \mathrm{EP} *)\end{array}$ \\
\hline 1 & 15 & 300 & $257,8 \pm 7,50$ & $52,1 \pm 4,52$ \\
2 & 15 & 250 & $186,5 \pm 9,05$ & $67,9 \pm 6,16$ \\
3 & 15 & 200 & $137,3 \pm 13,30$ & $76,7 \pm 11,32$ \\
4 & 14 & 150 & $62,4 \pm 12,31$ & $72,2 \pm 12,43$ \\
5 & 9 & 100 & $11,4 \pm 4,68$ & $34,7 \pm 4,05$ \\
6 & 3 & 50 & $0,00 \pm 0,00$ & $46,3 \pm 1,67$ \\
7 & 1 & 50 & $0,00 \pm 0,00$ & $50,0 \pm 0,00$ \\
\hline
\end{tabular}

*Erro padrão da média 
Tabela III. Número de larvas de Lysiphlebus testaceipes por Schizaphis graminum a $25 \pm 1^{\circ} \mathrm{C}, 60 \pm 10 \%$ de umidade relativa e 10 horas de fotofase.

\begin{tabular}{cccccc}
\hline \multirow{2}{*}{$\begin{array}{c}\text { Idade da fêmea } \\
\text { (dias) }\end{array}$} & $\begin{array}{c}\text { Número de fêmeas } \\
\text { (n) }\end{array}$ & \multicolumn{4}{c}{$\begin{array}{c}\text { Número de larvas do parasitóide por pulgão } \\
(\overline{\mathrm{X}} \pm \mathrm{EP} *)\end{array}$} \\
\cline { 3 - 5 } & & 1 & 2 & 3 & $4 \mathrm{a} 7$ \\
\hline 1 & 15 & $225,9 \pm 5,45$ & $13,1 \pm 2,91$ & $1,1 \pm 0,45$ & $0,6 \pm 0,38$ \\
2 & 15 & $160,7 \pm 5,21$ & $10,8 \pm 2,81$ & $1,0 \pm 0,62$ & $0,2 \pm 0,14$ \\
3 & 15 & $105,5 \pm 10,10$ & $9,4 \pm 2,55$ & $0,7 \pm 0,25$ & $0,6 \pm 0,22$ \\
4 & 14 & $58,6 \pm 11,50$ & $1,9 \pm 0,77$ & $0,0 \pm 0,00$ & $0,0 \pm 0,00$ \\
5 & 9 & $10,8 \pm 4,23$ & $0,3 \pm 0,33$ & $0,0 \pm 0,00$ & $0,0 \pm 0,00$ \\
6 & 3 & $0,0 \pm 0,00$ & $0,0 \pm 0,00$ & $0,0 \pm 0,00$ & $0,0 \pm 0,00$ \\
7 & 1 & $0,0 \pm 0,00$ & $0,0 \pm 0,00$ & $0,0 \pm 0,00$ & $0,0 \pm 0,00$ \\
\hline
\end{tabular}

*Erro padrão da média

Nos três primeiros dias de vida das fêmeas de L. testaceipes, cerca de $90 \%$ dos descendentes já haviam sido produzidos (Fig. 2). L. testaceipes apresentou uma fecundidade média de 646,7 ovos.

Foi verificado que, apesar de o suprimento de hospedeiros ter sido suficiente para o parasitóide L. testaceipes (Tabela II), ocorreu superparasitismo em cerca de $6 \%$ das ninfas de $S$. graminum (Tabela III). Já VAN SteEnIs (1994), quando determinou a tabela de vida de fertilidade de $L$. testaceipes em A. gossypii, observou apenas $1,34 \%$ de pulgões superparasitados. O autor sugere como explicação para o fato, que as fêmeas de L. testaceipes são incapazes de reconhecer um hospedeiro não parasitado de um recém-parasitado.

As fêmeas de L. testaceipes sobreviveram por até sete dias. No quarto dia observou-se que $93 \%$ ainda estavam vivas, e apresentaram uma longevidade média de 4,9 dias. No entanto, a partir de quatro dias houve uma diminuição gradativa da sobrevivência das fêmeas do parasitóide (Fig. 3).

Com relação aos parâmetros de crescimento populacional obtidos a partir da tabela de vida de fertilidade (Tabela IV), verificou-se que a taxa líquida de reprodução $\left(\mathrm{R}_{\mathrm{o}}\right)$ de $L$. testaceipes em $S$. graminum foi de 301,9; indicando que cada

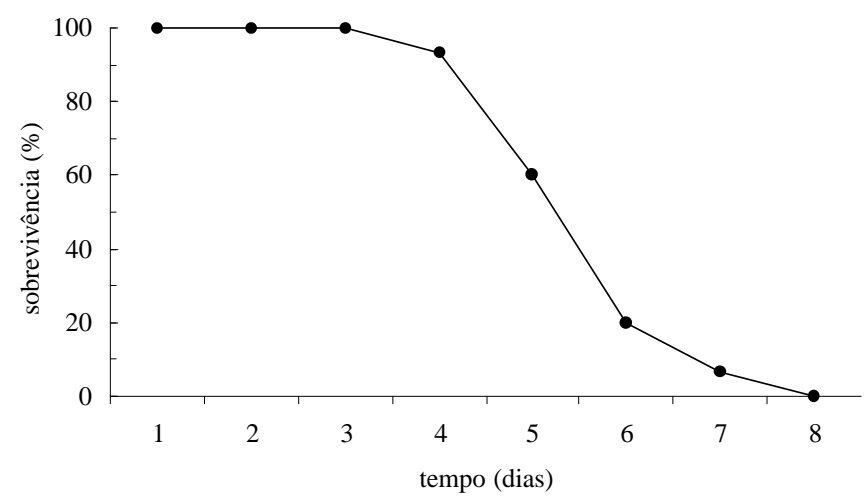

Fig. 3. Sobrevivência (\%) das fêmeas de Lysiphlebus testaceipes em Schizaphis graminum a $25 \pm 1{ }^{\circ} \mathrm{C}, 60 \pm 10 \%$ de umidade relativa e 10 horas de fotofase. fêmea, ao longo de sua vida, tem a capacidade de gerar cerca de 302 novos descendentes (Tabela V).

O principal dado que se obtém ao fazer-se uma tabela de vida de fertilidade é a taxa intrínseca de aumento $\mathrm{r}_{\mathrm{m}}$ (PEDIGO \& ZEISS 1996) e, segundo ANDREWARTHA \& BIRCH (1954), quanto maior o valor de $\mathrm{r}_{\mathrm{m}}$ mais bem sucedida será a espécie, em um determinado ambiente. Neste experimento, a taxa intrínseca de aumentar em número $\left(\mathrm{r}_{\mathrm{m}}\right)$ do parasitóide $L$. testaceipes em $S$. graminum foi 0,513 (Tabela V), sendo este valor superior ao encontrado por VAN STEENIS (1994) $\left(\mathrm{r}_{\mathrm{m}}=0,400\right)$ para essa mesma espécie de parasitóide, quando seu hospedeiro foi o pulgão $A$. gossypii. O afidiídeo Ephedrus californicus Baker, 1909 também apresentou uma taxa intrínseca inferior $\left(\mathrm{r}_{\mathrm{m}}=0,371\right)$ à observada para L. testaceipes, quando teve como hospedeiro Acyrthosiphon pisum (Harris, 1776) a $23^{\circ} \mathrm{C}($ COHEN \& MACKAUER 1987).

Dentre os diferentes atributos que um inimigo natural deve ter para que o mesmo seja capaz de controlar uma praga, a sua taxa intrínseca de aumento deverá, no mínimo, ser igual ao da

Tabela IV. Tabela de vida de fertilidade de Lysiphlebus testaceipes em Schizaphis graminum a $25 \pm 1{ }^{\circ} \mathrm{C}, 60 \pm 10 \%$ de U.R. e 10 horas de fotofase.

\begin{tabular}{cccc}
\hline $\mathrm{x}$ & $\mathrm{l}_{\mathrm{x}}$ & $\mathrm{m}_{\mathrm{x}}$ & $1_{\mathrm{x}} \mathrm{m}_{\mathrm{x}}$ \\
\hline 10,1 & 0,778 & 154,68 & 120,34 \\
11,1 & 0,778 & 111,90 & 87,06 \\
12,1 & 0,778 & 82,38 & 64,09 \\
13,1 & 0,727 & 37,44 & 27,22 \\
14,1 & 0,467 & 6,84 & 3,19 \\
15,1 & 0,156 & 0 & 0,00 \\
16,1 & 0,052 & 0 & \\
17,1 & 0,000 & & \\
\hline$\sum$ & & 393,24 & 301,90 \\
\hline
\end{tabular}

$\mathrm{x}=$ intervalos de idade (dias)

$1_{\mathrm{x}}=$ probabilidade de sobrevivência

$\mathrm{m}_{\mathrm{x}}=$ fertilidade específica

$1_{\mathrm{x}} \mathrm{m}_{\mathrm{x}}=$ número de fêmeas nascidas na idade $\mathrm{x}$ 
Tabela V. Parâmetros de crescimento populacional de Lysiphlebus testaceipes em Schizaphis graminum a $25 \pm 1{ }^{\circ} \mathrm{C}, 60 \pm 10 \%$ de umidade relativa e 10 horas de fotofase.

\begin{tabular}{ccccc}
\hline $\mathrm{R}_{\mathrm{o}}$ & $\mathrm{r}_{\mathrm{m}}$ & $\lambda$ & $\mathrm{T}$ & $\mathrm{TD}$ \\
(fêmeas) & (fêmeas/fêmeas/dia) & (fêmeas/dia) & (dias) & (semanas)
\end{tabular}

\begin{tabular}{lllll}
\hline 301,90 & 0,513 & 1,67 & 11,13 & 1,35 \\
\hline
\end{tabular}

$\mathrm{R}_{\mathrm{o}}=$ taxa líquida de reprodução

$\mathrm{r}_{\mathrm{m}}=$ taxa intrínseca de aumentar em número

$\lambda=$ razão finita de aumento

$\mathrm{T}=$ tempo médio entre gerações

$\mathrm{TD}=$ tempo de duplicação da população

praga em questão. GuLDEMOND et al. (1998) observaram que a taxa intrínseca de $A$. gossypii sobre crisântemo cultivar "White Reagan”, nas fases vegetativa, brotação nova e floração, foram, respectivamente, 0,$343 ; 0,323 ; 0,340$.

Assim, os resultados demonstraram que a taxa intrínseca do parasitóide L. testaceipes em S. graminum, em crisântemo, foi superior às taxas intrínsecas encontradas para o pulgão $A$. gossypii, por Guldemond et al. (1998), o que, segundo ANDREWARTHA \& BIRCH (1954), favorecerá o estabelecimento desse parasitóide em uma determinada área. Também, pode-se inferir que este afidiídeo atendeu a um dos critérios estabelecidos por VAN LeNTEREN (1986) o qual relata que, quando se deseja avaliar um inimigo natural visando utilizá-lo em liberações em ambientes protegidos, deve-se atentar para o fato de que o mesmo não será efetivo se sua capacidade intrínseca não for igual ou superior à da praga a ser controlada.

Quanto à razão finita de aumento (l) de $L$. testaceipes $\mathrm{em} S$. graminum, que é o fator de multiplicação da população original a cada intervalo unitário de tempo (ANDREWARTHA \& BIRCH 1954), esta foi de 1,67 por fêmea por dia (Tabela V). Isto demonstra a elevada capacidade de reprodução desse parasitóide em condições ideais de laboratório e tendo um hospedeiro adequado para sua reprodução. Contudo, no campo, é de se esperar que tal espécie esteja sujeita a diversos fatores ecológicos que podem alterar a sua capacidade reprodutiva. No entanto, quanto maior for a razão finita de aumento (l) maior será o número de indivíduos que serão adicionados à população.

O tempo médio entre o nascimento dos pais ao nascimento dos descendentes (T), ou seja, de uma geração de L. testaceipes, foi de 11,13 dias. Já o tempo para que ocorra a duplicação da população (TD) foi de 1,35 semana (Tabela V), indicando que esse afidiídeo tem condições de, em aproximadamente 10 dias, dobrar a sua população, o que é extremamente importante para uma criação massal tendo como hospedeiro $S$. graminum. Também no caso do uso de L. testaceipes como agente de controle biológico de pulgões, deve ser considerado que os pulgões são estrategistas "r", aumentando suas populações rapidamente, com superposição de gerações. No entanto, a infestação inicial de uma cultura por pulgões geralmente acontece através de um pequeno número e em focos isolados. Assim, a presença do parasitóide no início da infestação e um aumento também rápido de sua população, poderão prevenir surtos nas populações de pulgões e exercer o controle das mesmas.

Agradecimentos. Ao CNPq pelo apoio financeiro (Processo número 470705/01-9) e à CAPES e CNPq pela concessão das bolsas de estudo aos autores.

\section{REFERÊNCIAS}

Andrewartha, H. G. \& L. C. Birch. 1954. The innate capacity for increase in numbers, p. 31-54. In: H. G. Andrewartha \& L. C. Birch. (eds.). The distribution and abundance of animals. Chicago, University of Chicago Press, $x v+782$ p.

Bellows Junior, T. S.; R. G. Van Driesche \& J. S. Elkinton. 1992. Life-table construction and analysis in the evaluation of natural enemies. Annual Review of Entomology 37: 587-614.

Bergmann, E. C.; S. L. Imenes \& A. P. Takematsu. 1996. Pragas, p. 13-22. In: S. D. L. Imenes \& M. A. V. Alexandre (Coord.). Aspectos fitossanitários do crisântemo. São Paulo, Boletim Técnico do Instituto Biológico, $47 \mathrm{p}$.

Cohen, M. B. \& M. Mackauer. 1987. Intrinsic rate of increase and temperature coefficients of the aphid parasite Ephedrus californicus Baker (Hymenoptera: Aphidiidae). The Canadian Entomologist 119: 231-237.

ElLIot, N. C.; J. A. WebSTER \& S. D. KindLeR. 1999. Developmental response of Lysiphlebus testaceipes to temperature. Southwestern Entomology 24(1): 1-4.

Guldemond, J. A; W. J. Van Den Brink \& E. Den Belder. 1998. Methods of assessing population increase in aphids and the effect of growth stage of the host plant on population growth rates. Entomologia Experimentalis et Applicata 86: 163-173.

HÅgvar, E. B. \& T. Hofsvang. 1991. Aphid parasitoids (Hymenoptera, Aphidiidae): biology, host selection and use in biological control. Biocontrol News and Information 12(1): 13-41.

KRING, T. J. \& J. B. KRING. 1988. Aphid fecundity, reproductive longevity, and parasite development in the Schizaphis graminum (Rondani) (Homoptera: Aphididae)-Lysiphlebus testaceipes (Cresson) (Hymenoptera: Braconidae) system. The Canadian Entomologist 120(12): 1079-1083.

Mackauer, M.; J. P. Michaud \& W. VölKL. 1996. Host choice by aphidiid parasitoids (Hymenoptera: Aphidiidae): host recognition, host quality, and host value. The Canadian Entomologist 128(6): 959980.

Pedigo, L. P. \& M. R. Zeiss. 1996. Developing a degree-day model for predicting insect development, p. 67-74. In: L. P. PEDIGO. \& M. R. ZEISS. (eds.). Analyses in insect ecology and management. Ames, Iowa State University Press. xi+168.

Rodrigues, S. M. M.; V. H. P. Bueno \& J. S. de S. Bueno Filho. 2001. Desenvolvimento e avaliação do sistema de criação aberta no controle de Aphis gossypii Glover (Hem.: Aphididae) por Lysiphlebus testaceipes (Cresson) (Hym.: Aphidiidae) em casa-de-vegetação. Neotropical Entomology 30(3): 433-436.

Rodrigues, S. M. M. \& V. H. P. BuENo. 2001. Parasitism rates of Lysiphlebus testaceipes (Cresson) (Hym.: Aphidiidae) on Schizaphis graminum (Rond.) and Aphis gossypii Glover (Hem.: Aphididae). Neotropical Entomology 30(4): 625-629.

VAN Lenteren J. C. 1986. Parasitoids in the greenhouse: successes with seasonal inoculativa release systems, p. 342-374. In: J. WAAGE \& D. Greathead. Insect parasitoids. London, Academic Press, xvii+389 p.

Van Lenteren J. C. \& J. Woets. 1988. Biological and integrated pest control in greenhouses. Annual Review of Entomology 33: 239269.

VAN STEENIS, M. J. 1994. Intrinsic rate of increase of Lysiphlebus testaceipes Cresson (Hym.; Braconidae), a parasitoid of Aphis gossypii Glover 
(Hom., Aphididae) at different temperatures. Journal of Applied Entomology 118: 399-406.

VÖLKL, W.; D. H. STeChMAnN \& P. STaRÝ. 1990. Suitability of five Aphidiidae (Hymenoptera) for the biological control of the banana aphid Pentalonia nigronervosa Coq. (Homoptera, Aphididae) in the South Pacific. Tropical Pest Management 366(3): 249-257. 